\title{
A rare case of multiple fibroids with pregnancy
}

\author{
Jayesh R Shah ${ }^{1}$, Jiten Senta ${ }^{2}$, Shrina Shah ${ }^{3}$, Dhruv Patel ${ }^{4}$, Manish Pandya ${ }^{5, *}$, Kalpana Khanderiya ${ }^{6}$ \\ ${ }^{1}$ Associate Professor, C. U. Shah Medical College, Surendranagar, Gujarat, ${ }^{2,3,4}$ Resident, ${ }^{\mathbf{5}}$ Head of Department, Dept. of \\ Obstetrics \& Gynecology, ${ }^{6} \mathrm{HOU}$, Scientific Research Institute at Surendranagar, Gujarat, India
}

Corresponding Author:

Email: drmanish.pandya@gmail.com

\begin{abstract}
Leiomyomas are the most common benign tumor of the uterus and affect $40 \%$ to $50 \%$ of women older than $35 y$ rs of age. Usually they are asymptomatic( $70 \%$ to $80 \%$ ).approximately $5 \%$ of leiomyoma are of submucosal type being least common but usually are more symptomatic. symptomatic leiomayomas are managed with either medical therapy or surgical management in the form of myomectomy or hysterectomy. multiple submucosal fibroid in pregnancy is a rare and often complicate with a fetal morbidity and mortality ${ }^{1}$
\end{abstract}

Keywords: Fibroids, Miscarriage, Preterm labor, Fetal anomalies, Myomectomy, Hystrectomy, Uterine artery embolization.

\section{Introduction}

Fibroid tumor are benign tumor found in their 30's and 40's, fibroids are benign smooth muscle monoclonal neoplasms of uterus arising in the myometrium and sometime in the ligament attach. ${ }^{2}$

Uterine myoma usually asymptomatic during pregnancy. ${ }^{3}$ medical research has shown that the most uterine fibroids do not grow or shrink during pregnancy. It has been found that about a third of fibroids may enlarge during the first three months (first timester) of pregnancy. ${ }^{4}$ Studies have shown that the vast majority of women with fibroids have an uncomplicated pregnancy and childbirth. However it has been found that some women do unfortunately have some problems due to their fibroids. It is known that uterine fibroids are linked to a greater rate of spontaneous miscarriage, placental abruption, preterm labour, malpresentations, an abnormal or difficult labour need for caesarean section and intrapartum or postpartum haemorrhage. ${ }^{5}$

Making the diagnosis of fibroids during pregnancy is neither simple nor straightforward because the difficulty of differentiating fibroid from physiological thickening. ${ }^{6}$ Research has shown that only $42 \%$ of fibroid greater than $5 \mathrm{~cm}$ (labelled as large) and $12 \%$ of smaller fibroids (measuring 3-5 cm) can be felt and diagnosed on pelvic examination. Surprisingly, making the diagnosis using ultrasound is also difficult and this is mainly because of the difficulty of distinguishing fibroids from the normal thickening of the lining of the uterus (myometrium). It is therefore thought likely by specialists that the frequency of fibroids during pregnancy is actually underestimated. ${ }^{7}$

Myomectomy is most commonly performed procedure during pregnancy with fibroid at a time of caesserian section. ${ }^{8}$ Surgical management of leiomyoma during pregnancy (intrapartum) is safe in carefully selected patients. ${ }^{9}$

\section{Case Report}

A 48years old female came to opd with complaints of heavy bleeding per vaginal associated with lower abdominal pain. Her last menstrual period date $26 / 12 / 2017$, regular, cyclic, heavy flow, she is nulligravida and underwent for diagnostic dilatation \& curretage 15 years back. She was married at 16 years of age and her active married (aml) life of 11years and divorced after that because of she never conceived during aml period. During that period there is history of single in vitro fertilization cycle and outcome was negative, she married again. During her $2^{\text {nd }}$ aml no positive history of pregnancy both husband are fertile.
On examination pulse: 110/min,bp$130 / 80 \mathrm{mmhg}$,respiratory rate-17/min,spo2-98\% local examination 20-24 weeks size of uterus, bulky, mobile, fornices clear, bleeding present.

Usg: multiple uterine fibroids of variying size $(7 \times 5,6 \times 5,7 \times 6,3 \times 2)$ with live foetus in utero of 12 weeks of gestational age fetal heart rate-150/min.as shown in (Fig.1, 2, 3, 4, 5)

Investigation: (blood \& urine)hb-9.10 $\mathrm{mg} / \mathrm{dl}$,wbc9850,platelets:- ,BGRH -O POSITIVE- ,HIV-non reactive, $\mathrm{HBsAg}$-non reactive, creatinine- $0.58 \mathrm{mg} / \mathrm{dl}$, urea- $16.2 \mathrm{mg} / \mathrm{dl}$, rbs- $82.40 \mathrm{mg} / \mathrm{dl}$, urine routine-PUS CELL:55-60Ccell/hpf, Epithelial cell-10-15 cell/hpf, Red blood cell-absent, Cast -absent, Crystals-cal. oxalate(occ).

Ecg: Within normal limits.

Chest x-ray: Both lungs fields are normal both $\mathrm{cp}$ angels well defined cardiac shadow appears normal. 


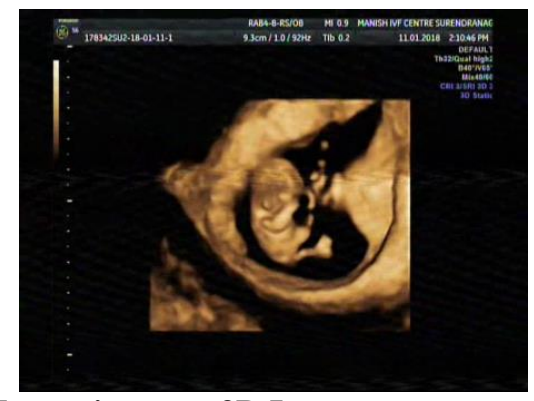

Fig. 1: Foetus in utero 3D Image

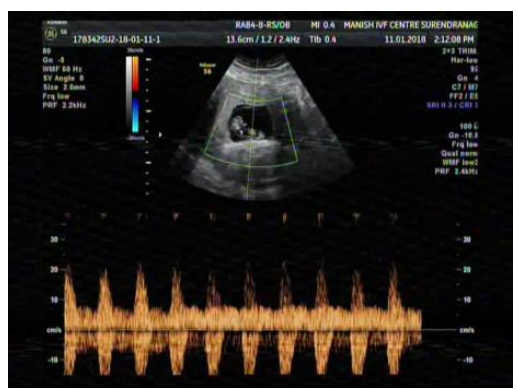

Fig. 2: FHR of the foetus

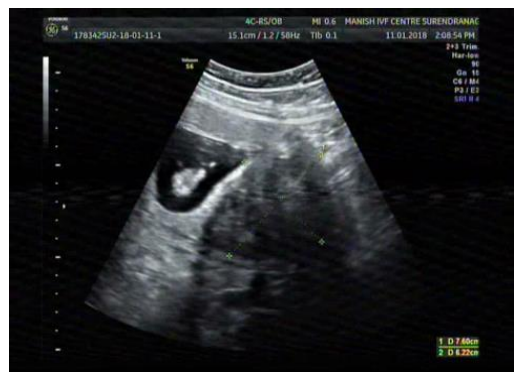

Fig. 3: Fibroid with foetus

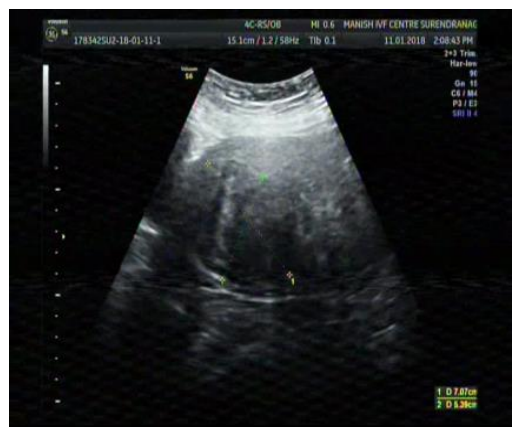

Fig. 4: Measuring size of fibroid

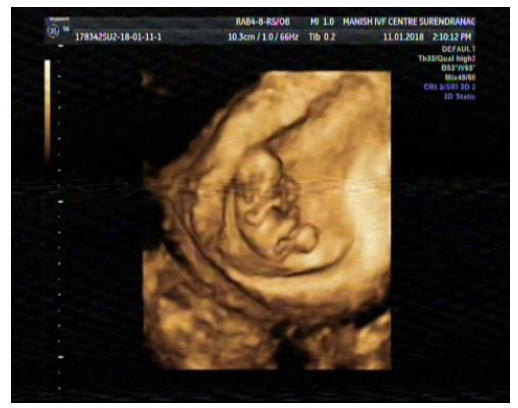

Fig. 5: 3d image of foetus

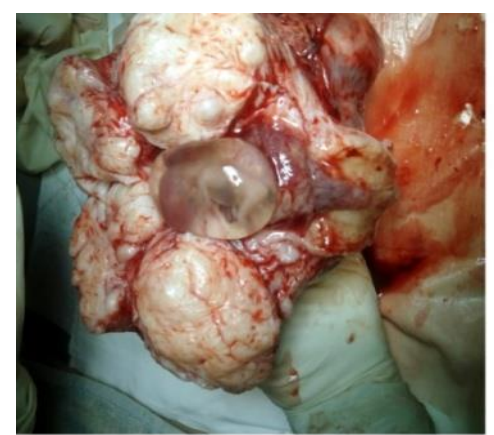

Fig. 6: Cut section showing multiple

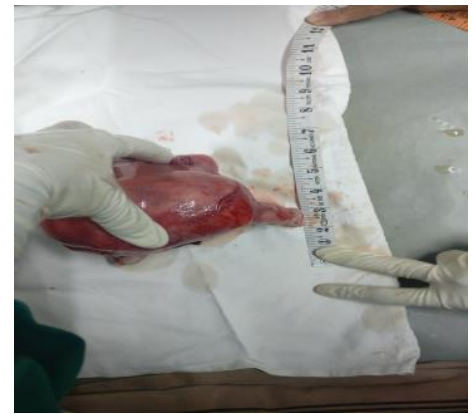

Fig. 7 Specimen of uteus

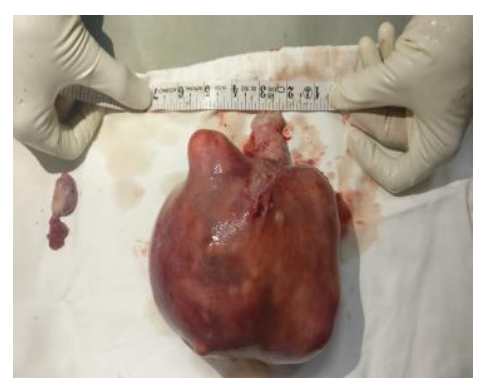

Fig. 8: Specimen of uteus

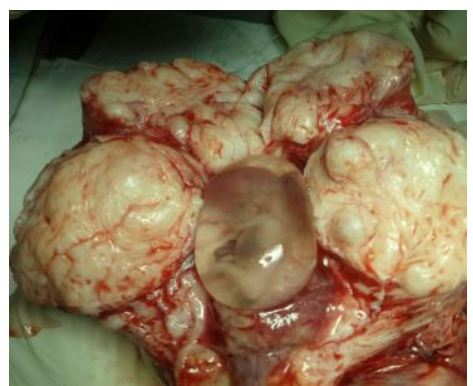

Fig. 9: Specimen of uteus

\section{Management}

Multiple fibroids with pregnancy and bleeding per vaginum leading indication for hysrectomy as doctor point of view. She is feeling uncomfortable after knowing that she is pregnant, though pregnancy is accidental finding during examination and was nulligravida and counseled by us and family members to continue the pregnancy. After discussion with the family members and her husband its turn out that she don't want to continue the pregnancy as this is her $2^{\text {nd }}$ 
marriage and she is mother (children's from $1^{\text {st }}$ marriage of his husband), and having grandson so due to personal reasons she don't want to continue the pregnancy, so final decision is pannhystrectomy (according to age and prevent further complication after reserving ovaries and fallopian tube).

Pre-operative: Injectable antibiotics given for 2days for infection and one unit of whole blood given.

Operative: PANHYSTRECTOMY performed under spinal anesthesia.as shown in Fig. 6,7,8,9

Post-operative: One unit of whole blood given injactable antibiotic for 3 days and shifted to oral antibiotics for 5 days with analgesics $\&$ antacid.

\section{Discussion}

Fibroids are very common in women of reproductive age. Most fibroids do not grow (or become smaller) in pregnancy, but about 30\% may enlarge during the first three months of pregnancy. The results from studies are somewhat conflicting, but it is reassuring to note that the majority of women with uterine fibroids will have uncomplicated pregnancies and childbirth.

Uterine fibroids are linked to an increased rate of spontaneous miscarriage, preterm labour, foetal malpresentation, labour dystocia, caesarean section and postpartum haemorrhage, hystrectomy. ${ }^{10}$

The most frequent complication of a fibroid in pregnancy is pain. The symptoms can generally be managed with conservative treatment, but very rarely surgical treatment is necessary. A caesarean section may be advisable for those women who have previously had a myomectomy, especially if the cavity of the uterus was opened. Uterine artery embolization is a reasonable alternative treatment option to surgery in the event of fibroids causing severe problems, but uerine artery embolissation is absolutely contra-indicated during pregnancy and in those women who wish to fall pregnant in the future. Those don't want to fall pregnant in future hysrectomy is advisable, hystrectomy is indicated for uterine fibroids that cause substantial bleeding, pelvic pain or pressure or anemia refractory to iron replacement in women not wishing to preserve fertility. ${ }^{11}$

\section{Conclusion}

A 48years old female came to opd with complaints of heavy bleeding per vaginal associated with lower abdominal pain. On usg finding she diagnosed as a multiple fibroids with pregnancy, she is already grandmother and she don't want any further child so for this patient we planned for panhysterectomy.

\section{References}

1. Spontaneous expulsion of uterine fibroid vaginally; mimicking inevitable abortion: A case report Kamal Singh,*, Sita Thakur, Indu Saroha. Lecturer, Professor, Junior resident, department of obstetrics and gynaecology, Dr Rajender Prasad Govt Medical College Kangra at Tanda Himachal Pradesh, India.

2. Modern gynecology, Ajit Virkud, $3^{\text {rd }}$ edition 2017 page 115.

3. Annachiara basso, Mariana Rita Catalano, ${ }^{1}$ Giuseppe Loverro, Serena Nocera, Edoardo Di Naro, Matteo Loverro, et al uterine fibroid torsion during pregnancy: A case of laparotomic myomectomy at 18 weeks' gestation with systematic review of the literature.

4. Management of uterine fibroids in pregnancy: Recent trends.

5. Itale sg, Padula F, Gulino Fa, Fibroids in pregnancy-common but poorly understood.

6. Hee joong lee, Md, phd, Errol $\mathrm{r}$ norwitz, Md, Phd, and Julia S, MBA haw, Md. Contemporary management of fibroids in pregnancy.

7. Muram d, Gillieson m, Walters JH. Myomas of the uterus in pregnancy: Ultrasonographic follow-up.

8. Bajekal n, li Tc fibroids, infertility and pregnancy wastage.

9. Burton $\mathrm{CA}^{1}$, grimes da, march cm. Surgical management of leiomyomata during pregnancy.

10. Rice JP, Kay HH, Mahony BS. The clinical significance of uterine leiomyomas in pregnancy.

11. Karen J. Carlson, David h. Nichols, and Isaac Schiff. Indications for hysterectomy. 